\title{
Power Management for Throughput Enhancement in Wireless Ad-Hoc Networks
}

\author{
Tamer A. ElBatt ${ }^{+}$, Srikanth V. Krishnamurthy*, Dennis Connors* and Son Dao*1 \\ ${ }^{*}$ HRL Laboratories, LLC, 3011, Malibu Canyon Road, Malibu, CA 90265 \\ E-Mail: krish@hrl.com, connors@wins.hrl.com, skdao@hrl.com \\ ${ }^{+}$University of Maryland, College Park, MD 20742 \\ E-Mail: telbatt@eng . umd.edu
}

\begin{abstract}
In this paper we introduce the notion of power management within the context of wireless ad-hoc networks. More specifically, we investigate the effects of using different transmit powers on the average power consumption and end-to-end network throughput in a wireless ad-hoc environment. This power management approach would help in reducing the system power consumption and hence prolonging the battery life of mobile nodes. Furthermore, it improves the end-to-end network throughput as compared to other ad-hoc networks in which all mobile nodes use the same transmit power. The improvement is due to the achievement of a tradeoff between minimizing interference ranges, reduction in the average number of hops to reach a destination, the probability of having isolated clusters, and the average number of transmissions (including retransmissions due to collisions). The protocols would first dynamically determine an optimal connectivity range wherein they adapt their transmit powers so as to only reach a subset of the nodes in the network. The connectivity range would then be dynamically changed in a distributed manner so as to achieve the near optimal throughput. Minimal power routing is used to further enhance performance. Simulation studies are carried out in order to investigate these design approaches. It is seen a network with such a power managed scheme would achieve a better throughput performance and lower transmit power than a network without such a scheme.
\end{abstract}

\section{INTRODUCTION}

Power based connectivity definition is a new concept in wireless ad-hoc networks. It attempts to improve the endto-end network throughput and the average power consumption. This is due to the fact that as the power gets higher, and the connectivity range increases, each node would reach almost all other nodes in a single hop. However, since higher powers cause a higher interference level, more collisions occur, and hence there will be more transmission attempts. By reducing the transmission power levels at each node such that the node can directly connect

\footnotetext{
1“This work was done at HRL Laboratories, LLC."
}

to only a small subset of nodes in the network, the interference zones are considerably reduced. However, under this proposition a packet has to be relayed by many intermediate nodes in order to reach the destination. Since there is a large number of transmissions, throughput may again degrade due to the increase in interference. Our protocol attempts to dynamically reach a near-optimal power level such that the network throughput is brought close to the maximum achievable throughput. This also translates to reducing the total power usage to a level close to the minimum. The major advantage of our approach is power saving, since power is a precious resource in the wireless environment. Moreover, this will lead to improving the throughput as well. Typical networks that might benefit from the concept of power based routing are low mobility (typically pedestrians) wireless ad-hoc networks that need to be established for soldiers relaying information for situational awareness on the battlefield, rescue and emergency disaster relief operations.

Various routing algorithms have been proposed for wireless ad-hoc networks in the literature. Those algorithms are mainly focused on establishing routes, and maintaining these routes under frequent and unpredictable connectivity changes [2], [3]. The implicit assumption in most of the earlier work is that nodes' transmitted powers are fixed. To the best of our knowledge, there is no prior known work that proposes the concept of mobile ad-hoc nodes using different transmit powers. It is evident that this approach is restricted to ad-hoc networks of relatively low mobility patterns. If the nodes are highly mobile, the power management algorithm might fail to cope with the fast and sudden changes due to fading and interference conditions. In [1], Bambos refers to power control as being widely accepted in the context of cellular (both channelized and CDMA) systems and satellite systems. On the other hand, he refers to the limited attention that power control has received in mobile ad-hoc networks. This work investigates the benefits, and possibly the tradeoffs, of deploying dif- 
ferent transmit powers in the wireless ad-hoc environment. We propose a power management scheme which can be used in conjunction with traditional table-driven routing protocols, with possibly minor modifications. The performance measures are taken to be the end-to-end network throughput and the average power consumption.

The paper is thus organized as follows: In section II, the system model is introduced. The connectivity range optimization is introduced in section III. The simulation results are given and discussed in section IV. In section V, possible protocol implementations are discussed. Finally, the conclusions are drawn in section VI.

\section{System Model}

When the power management scheme is implemented, each node transmits at a minimum power level such that only a fixed number of neighboring nodes can hear the transmission. For example, a node might transmit with a power such that only its three closest neighbors can hear its transmission. Thus, in Figure 1 below, node A transmits with a power $P_{1}$ such that only it's three nearest neighbors i.e., nodes B, C and D can hear it. Similarly, node D would transmit with a different power, say $P_{2}$, such that only it's three nearest neighbors i.e., nodes $\mathrm{A}, \mathrm{C}$ and $\mathrm{E}$ can hear it.

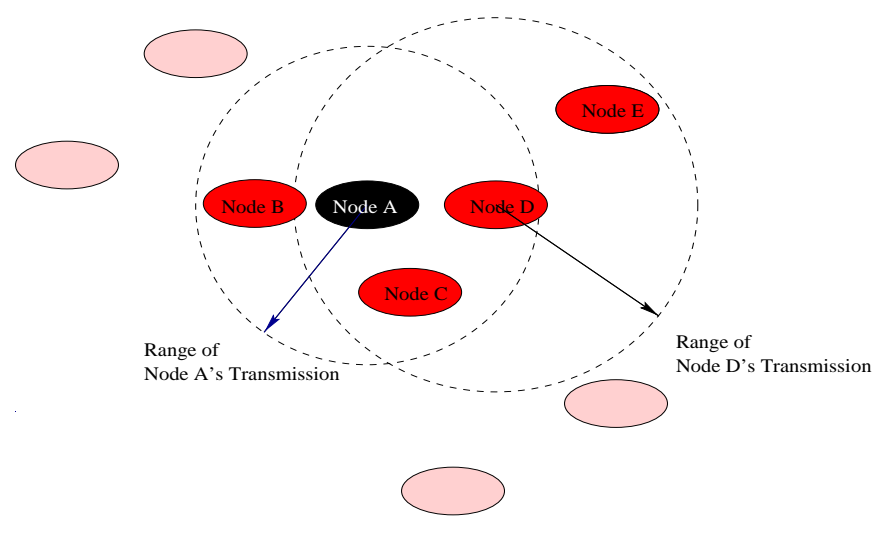

Figure 1: A Power-Controlled Ad-Hoc Network

In order to set up the framework to investigate the effect of transmit powers on the end-to-end network throughput, we make the following assumptions and introduce some appropriate notations:

1. The wireless ad-hoc network consists of $\mathrm{n}$ nodes; each node has a unique ID, denoted by Node ID.

2. The mobile nodes are assumed to have low mobility patterns, that is, they are typical pedestrians. This, in turn, implies that the network topology changes slowly and the class of shortest-path routing algorithms is applicable.
3. Each mobile node has direct connectivity to its $N$ closest neighbors only, where $N$ is to be adapted dynamically. 4. Assume connectionless (datagrams) type of traffic, i.e. routing decisions are made on a packet-by-packet-basis.

5. The transmit power of any mobile node is upper bounded by a maximum power level denoted as $P_{\max }$. The limited size and weight of the mobile terminal dictate this constraint.

6. The transmit power of any mobile node is lower bounded by a minimum power level $P_{\text {min }}$. This constraint is essential to avoid partitioning the network into isolated islands.

7. Two MAC schemes are deployed in this system. A contention-free MAC scheme is employed during connectivity setup. In addition, we employ a classical SlottedAloha MAC scheme during data transfer, (any contentionbased scheme may be chosen).

8. This protocol assumes the availability of a reliable reverse channel that operates in a different frequency band. This channel is essential for performing the following tasks:

- Sending the $A C K$ and $N A C K$ messages from the receiving node to the transmitting node in order to retransmit packets involved in collisions.

- If a data packet reaches its destination successfully, the destination node is expected to broadcast an acknowledgement message, at possibly the maximum allowable power level, in order to reach the source node. This acknowledgement enables each node to periodically compute its end-to-end throughput which is to be optimized. The protocols will use this computation in order to drive the average throughput towards a maximal value.

9. Guard bands are crucial in order to keep the nodes in the network time-synchronized. More specifically, the slot duration is assumed to be larger than packet duration by interval equal to a guard band. These bands are needed to compensate for arbitrary delays incurred by transmitted packets due to signal propagation delays or clock drifts.

10. We assume that each mobile node has two buffers:

- MAC Buffer: This buffer is required in order to store packets arriving during a time slot until the beginning of the next slot. When the buffer is full, packets are dropped and they are treated as lost packets.

- Retransmission Buffer: This buffer stores transmitted packets, temporarily, until it receives a message from the next node. If it receives an $A C K$ message, it discards the packet. On the other hand, if it receives an NACK message, it retransmits the packet after a random period of time.

11. We deploy the classical shortest-path routing algorithm with a slight modification. The link costs are chosen 
to be the transmitted powers. Therefore, the objective is to route the packet from the source to the destination through the minimum power path.

12. The received power at any mobile node has to be greater than a minimum power level, denoted by MinRecvPower. This is crucial in order to guarantee reliable communication between the transmitter and the receiver. This value helps determine the power level at which a mobile has to transmit in order to directly reach a neighboring node.

13. It is expected that unidirectional links will be formed when transmit powers are thus manipulated, i.e. we might have a cluster of nodes that can communicate with each other but no packets can either enter or leave this cluster. Modifications to the the protocol to eliminate this effect are being investigated. However, it should be noted that this does not change the routing methodology ${ }^{1}$ since the signaling channel is bidirectional.

14. The Signaling Packet format is shown in Figure 2 below,

\begin{tabular}{|l|l|l|}
\hline Node ID & Neighbor ID & Transmit Power Level \\
\hline
\end{tabular}

Figure 2: Signaling Packet Format

where,

Node ID: Identifier for the node broadcasting the signaling packet.

Neighbor ID: Identifier for a direct neighbor to which the node is broadcasting the signaling packet.

Transmit Power Level: Minimum power level needed to reach that neighbor.

15. The Data Packet format is shown in Figure 3.

\begin{tabular}{|l|c|l|}
\hline Source ID & Destination ID & Current Node ID \\
\hline Next Node ID & Re-Transmissions & Payload \\
\hline
\end{tabular}

Figure 3: Data Packet Format

where,

Source ID: Identifier of the node that generated the packet. Destination ID: Identifier of the packet's destination node. Current Node ID: Identifier of the relay node at which the packet is currently stored on its path to the destination.

Next Node ID: Identifier of the next relay node to which the packet is to be transmitted on its path to the destination.

\# Re-Transmissions: Total number of retransmission attempts performed on that packet. (retransmission will be

\footnotetext{
${ }^{1}$ Table driven routing is still feasible.
}

necessary whenever a packet encounters a collision)

16. The Connectivity Table, for the wireless ad-hoc network shown in Figure 1, is suggested to have the format shown in Figure 4.

\begin{tabular}{|l|l|l|}
\hline Node ID & Neighbor ID & Transmit Power Level \\
\hline A & B & $P_{1}$ \\
\hline A & C & $P_{1}$ \\
\hline A & D & $P_{1}$ \\
\hline D & A & $P_{2}$ \\
\hline D & C & $P_{2}$ \\
\hline D & E & $P_{2}$ \\
\hline
\end{tabular}

Figure 4: Connectivity Table Format

17. Node Throughput is defined as percentage of successful transmission attempts.

18. End-to-End Network Throughput is defined as percentage of packets that reach their destinations successfully and is denoted by $\eta$.

19. Average Power Consumption is defined as average transmitted power/node/slot and is denoted by $\bar{P}$.

20. The channel model includes only path loss and shadowing effects. We assume the lognormal random variable $\zeta$ to depict shadowing. Thus, the received power is given by,

$$
P_{r}=\frac{\zeta}{d^{4}} \cdot P_{t}
$$

where,

$P_{t}$ : power transmitted.

$\mathrm{d}$ : distance between the transmitter and the receiver.

\section{Connectivity RAnge Optimization}

\section{A. Problem Formulation}

Considering various conditions of connectivity and power management, it is straightforward to point out the following issues:

- Consider a wireless ad-hoc network with all mobile nodes using the maximum power level (i.e. no power management). Accordingly, any mobile node can reach a large number of nodes in just one hop. The advantage of this approach is reaching a large number of nodes in a single hop and almost all of the nodes in the network in two hops. The price paid is however twofold, namely high power consumption and higher interference, which results in a large number of collisions. If the link cost is taken to be the transmitted power, it is straightforward to notice that the 
cost of all the links are equal $\left(=P_{\max }\right)$. Hence the minimum power routing reduces to the minimum hop routing. This case serves as a reference case for comparison purposes.

- We next consider a wireless ad-hoc network, consisting of $n$ nodes, with a connectivity range of $N$, where $2 \leq N$ $<n$-1. Each mobile node has a direct link to the closest $N$ out of (n-1) mobile nodes. We call these $N$ nodes a cluster. Given $N$, the mobile node adjusts its power to reach at most the farthest node within its cluster. However, we assume that there is no power adaptation within the cluster. The advantages of this approach are lower power consumption and possibly, a node's transmission will cause lower interference to other simultaneous transmissions, when compared to the previous case. The drawbacks are a higher number of hops might have to be traversed in order to reach a destination, and there exists the possibility of having isolated clusters. Note that link costs (transmitted powers), in this context, are generally different depending on the radius of each cluster. Accordingly, incorporating the minimum power routing algorithm is crucial to limit power consumption.

Limitation: Since there is no power adaptation within a cluster, it is possible that a mobile node communicates with a node within its cluster using a power level higher than the minimum required power to communicate with that node, and thereby possibly introduces more interference than that incurred in the case to be discussed next.

- Finally, we consider a wireless ad-hoc network with connectivity $N$, where $2 \leq N<n-1$. Again, each mobile node has a direct link to the closest $N$ (out of $(n-1)$ ) mobile nodes. We assume, in this case, that there is power adaptation within the cluster. This approach reduces the powers consumed on various routes. Thus, in order to communicate with another node, a node would use the minimum power that guarantees reliable communication with that node. Note that this approach would minimize the interference caused by such a transmission. The advantages and drawbacks are the same as in the previous case. We would expect this approach to outperform (achieve higher throughput) the previous scheme at the expense of higher complexity. The minimum power routing is once again the candidate routing algorithm.

Consider the third case described above. Our objective is to solve the following minimization problem:

$$
\min _{N}(-\eta+\alpha \cdot \bar{P})
$$

s.t.

$$
P_{\min } \leq P_{t_{i}} \leq P_{\max }
$$

where,

$P_{t_{i}}$ is the transmitted power of node $i$, and $\alpha$ is the fixed weighting factor that reflects the relative importance of the two components of the afore mentioned composite cost function.

The choice of the parameter $\alpha$ is rather arbitrary, and there is no well-defined procedure for choosing it. The following formulation is equivalent and much easier to implement,

$$
\max _{N} \eta
$$

s.t.

$$
\begin{gathered}
\bar{P} \leq \beta \\
P_{\text {min }} \leq P_{t_{i}} \leq P_{\text {max }}
\end{gathered}
$$

where $\beta$ is the equivalent parameter and has a one-to-one correspondence to $\alpha$.

\section{B. System Operation}

Each mobile node is responsible for keeping track of its closest neighbors (in terms of transmitted power) and updating its local connectivity tables accordingly. The time taken to update the network topology has to be small in comparison with the time between location updates. In the following descriptions, we briefly describe the functions performed at each mobile node:

1. Each mobile node is assigned a dedicated signaling time slot of a global signaling channel. In this slot, the node is allowed to broadcast a beacon packet, using the $P_{\max }$ level, to all other nodes in the area of interest. Note that the MAC protocol employed for signaling slot assignment supports contention-free communications, and hence no collisions occur in this phase.

2. In slot $i$, all other nodes obtain the beacon signal of node $i$. Accordingly, they record the received power level and store it in a data structure along with the mobile node's ID ${ }^{2}$. Using a set consisting of the latest, predetermined number of received power level measurements, an average is computed. Note that average power measurements are used, rather than instantaneous power measurements. This is motivated by the fact that average power measurements smooth out variations due to fast multipath fading, which

\footnotetext{
${ }^{2}$ This functionality is simulated in our model using the Power Measurement procedure described later in this section.
} 
is not compensated for by this scheme.

3. By the end of this phase, mobile node $i, 1 \leq \mathrm{i} \leq \mathrm{n}$, is expected to have a ranking of all other nodes and this ranking is based on the average received power levels from those nodes. Based on this ranking, node $i$ picks its $N$ closest nodes (having highest average received power levels at this node's site) as its direct neighbors. Subsequently, node $i$ updates its local connectivity table by adding the mobile node IDs of its direct neighbors.

4. Each node then adapts its transmit power level in order to achieve the required connectivity, i.e. direct links are established only to the closest $N$ nodes.

5. Node $i$ updates its local connectivity table in order to store the link costs to the direct neighbors. The link cost in this protocol is taken to be the transmit power level.

6. Each node then broadcasts a Signaling Packet containing its local connectivity table information in the signaling channel. Thus, each mobile node obtains and then stores the global network topology information. This information is then used in forming its local routing table. Note that a global topological view is essential for the functioning of the table-driven routing algorithms. For large networks, it is not feasible for each node to store the entire global topological information due to the heavy communication overhead incurred and also due to memory constraints. Accordingly, this scheme supports small to midsize wireless ad-hoc networks or subnetworks of a large ad-hoc network.

\section{B.1 Power Measurement}

This procedure emulates the operation of mobile node $j$ capturing the beacon signal transmitted by node $i$ during node $i$ 's allocated signaling slot, where $l \leq i, j \leq n$ and $i \neq j$. The received signal strength depends solely on the transmitted power level (which is assumed to be $P_{\max }$ during this phase), the current positions of nodes $i$ and $j$, and the effect of the log-normal shadowing. Thus, the received power level is computed by using the following formula:

$$
P_{r_{j i}}=\frac{\zeta}{d_{j i}^{4}} \cdot P_{t_{i}}
$$

where,

$P_{r_{j i}}=$ received power level at node $j$ from node $i$.

$\mathrm{P}_{t_{i}}=$ transmitted power by node $i$.

$d_{j i}=$ current distance between node $j$ and node $i$.

$\zeta=$ log-normal shadowing coefficient.

As pointed out earlier, we rely on average power measurements rather than instantaneous power measurements. This is due to the fact that instantaneous measurements could be inaccurate in reflecting the slowly varying chan- nel conditions in the presence of fast multipath fading. Therefore, a moving average is computed by each node to average out the fast fading over a pre-specified number of most recent instantaneous power measurements.

\section{B.2 Power Management}

There are two suggested approaches for power management in mobile ad-hoc networks:

\section{- No power adjustment within a cluster.}

- Power adjustment within a cluster.

The basic difference between the two schemes is that in the former scheme, the power needed to communicate with the farthest node in the cluster is also used to communicate with any closer node in the cluster. On the other hand, the latter scheme suggests communicating with each node using the minimum power it needs for reliable communication. This introduces less interference to simultaneous transmissions of other nodes.

The objective of defining a cluster is to reduce collisions/interference and thereby improve the end-to-end network throughput. As mentioned earlier, we assume a minimum required level of received power, denoted MinRecvPower, that is necessary to guarantee a maximum accteptable bit error rate. The minimum power level to be transmitted by node $i$ such that at least the MinRecvPower level is achieved at node $j$ for a given network configuration is given by:

$$
P_{t_{i j}}=P_{m a x} \cdot \frac{\text { MinRecvPower }}{P_{r_{j i}}}
$$

where,

$P_{t_{i j}}=$ power transmitted by node $i$ such that the transmission range does not exceed node $j$.

$P_{t_{j i}}=$ power received by node $j$ when node $i$ transmits at $P_{\max }$ for the given configuration.

\section{B.3 Minimum Power Routing}

The Minimum Power Routing (MPR) algorithm proposed is a hop-by-hop shortest path routing mechanism where the link costs are the transmitted power levels.

The routing algorithm then goes through the following steps:

1. Based on the routing table constructed, the mobile node creates the set of all possible routes from the source to destination.

2. The routing algorithm employed falls within the general class of shortest path routing. It searches, within the created route set, for the minimum cost route from source to destination.

3. Determine the next relay node on the minimum power 
route.

4. Modify the Next Node ID field in the data packet being routed.

5. Copy the packet to the retransmission buffer until its successful reception at the next node is indicated via an $A C K$ message.

6. The packet is sent to the MAC module for transmission to the next relay node.

\section{Mobility Model}

The power management based routing is recommended for networks characterized by low mobility patterns, namely pedestrians. In this section, we present the mobility model employed in the proposed system. The position of each node is updated periodically, every certain number of seconds. The new position is determined using the current position coordinates, the speed of the mobile node, and the direction of motion. The speed of the mobile is drawn from a random variable uniformly distributed between minimum and maximum values. Moreover, the direction of motion is assumed to be uniformly distributed between $[0$, $2 \pi]$.

\section{Simulation Results}

We developed an OPNET based simulation model for a wireless ad-hoc network that consists of 25 nodes. Due to memory and simluation time constranits, we set the value of $P_{\max }$ such that the transmission range of any node using $P_{\max }$ spans at least 15 out of the 25 nodes in the network. Therefore, the connectivity range $N$ was limited to take values between 2 and 15 as reflected in Table 1. The threshold MinRecvPower was chosen to be 1 milliwatt. We assume that this would be sufficient and would guarantee a minimum acceptable bit error rate at the receiver. Note from Table 1 that the mobility model parameters were chosen to reflect the low mobility pattern being considered.

Table 1. System Paramters

\begin{tabular}{|l|l|}
\hline $\mathrm{n}$ & $25 \mathrm{nodes}$ \\
\hline $\mathrm{N}$ & $2,3,4, \ldots . ., 15$ \\
\hline$P_{\max }$ & $100 \mathrm{mw}$ \\
\hline MinRecvPower & $1 \mathrm{mw}$ \\
\hline Packet Arrival Rate/node & $0.05,0.1,0.2 \mathrm{pkts} / \mathrm{sec}$ \\
\hline Frame Duration & $25 \mathrm{msec}$ \\
\hline Slot Duration & $2 \mathrm{msec}$ \\
\hline Minimum Speed & $1 \mathrm{~m} / \mathrm{sec}$ \\
\hline Maximum Speed & $5 \mathrm{~m} / \mathrm{sec}$ \\
\hline Time between location updates & $10 \mathrm{sec}$ \\
\hline Simulation Time & $800 \mathrm{sec}$ \\
\hline
\end{tabular}

Our main objective is to investigate the impact of manipulating the Connectivity Range $N$ on the end-to-end network throughput and on the average power consumption. We first consider the "no power adjustment within a cluster" approach. In Figure 5, the the average node throughput is plotted versus $N$ for different network loads. It is noticed that the average node throughput decreases as the connectivity range $N$ increases. Note that when $N$ increases, more nodes compete for transmitting in the same time slot, and hence collisions become more likely. However, when $N$ increases, packets are expected to traverse fewer number of hops to destination, which implies that fewer intermediate nodes attempt transmissions in the same slot. The results seem to imply that the advantage gained by the latter effect does not offset the disadvantage of the increased interference and collisions due to the former effect. In our simulations, Destination IDs were generated according to a uniform distribution. This, in turns, reduces the effect of the second factor, since the number of hops travesred from source to destination depends mainly on the distance between those nodes. Thus, the first factor may be expected to dominate the behavior of the node throughtput as $N$ increases. On the other hand, if the Destination node was restricted to be sufficiently far from the source node, we would expect the contribution of the second factor to be more, and in this scenario, we might expect it to offset the first factor. In Figure 6, the end-to-end network through-

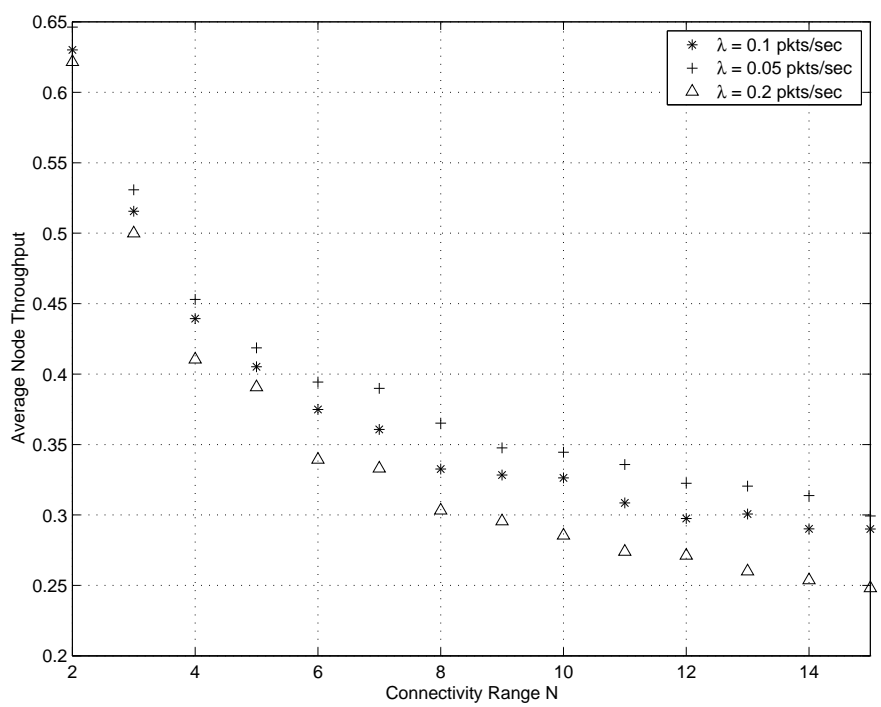

Figure 5: Average Node Throughput

put is shown for different network loads. Notice that the throughput achieved is relatively low due to the underlying slotted aloha MAC protocol. However, they are useful in comparing various design alternatives that provide insight about various design tradeoffs. It can be noticed that the maximum end-to-end throughput is achieved for val- 
ues of $N$ less than 15 (which corresponds to the no power management case). This implies that including a transmit power control/management scheme in a wireless ad-hoc environment improves the network throughput. In Figure 7 , the average power consumption is plotted versus $N$ for different network loads. The average power consumption increases monotonically as $N$ increases. Again, this can be explained due to the afore mentioned opposing factors affecting the average node throughput. As in the case of the node throughput, the first factor dominates the average power consumption behavior as $N$ increases.

In Figures 6 and 7, we can observe, for $\lambda=0.1$ packets/sec, the trade-offs between the end-to-end throughput and the average power consumption. For $2 \leq N \leq 9$, it is noticed that that in order to reduce average power consumption, the end-to-end throughput has to be sacrificed. In (3), if we impose the following constraint on $P_{a v}$ :

$$
P_{a v} \leq 50
$$

it is seen from Figure 6 that the maximum achievable endto-end network throughput is $0.32{ }^{3}$ Notice from Figures 5,6 , and 7 that the same trends are preserved for a variety of network loads.

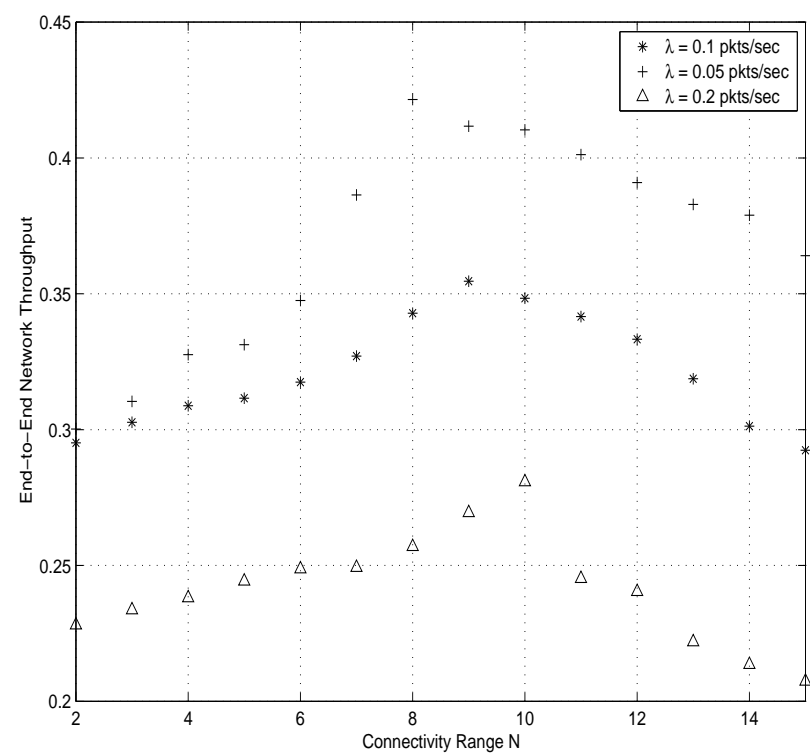

Figure 6: End-to-End Network Throughput

Next, we consider the "power adjustment within a cluster" approach. As explained earlier, each node can communicate witH any other node if the power at the receiving node is larger than the minimum received power needed for reliable communication. Therefore, this approach is expected

\footnotetext{
${ }^{3}$ This is for the particular value of the packet arrival rate. Note that by definition, the end-to-end throughput is the percentage of the total transmitted packets that actually reach their destinations. This definition of throughput is different from the traditional definitions of throughput for slotted aloha systems.
}

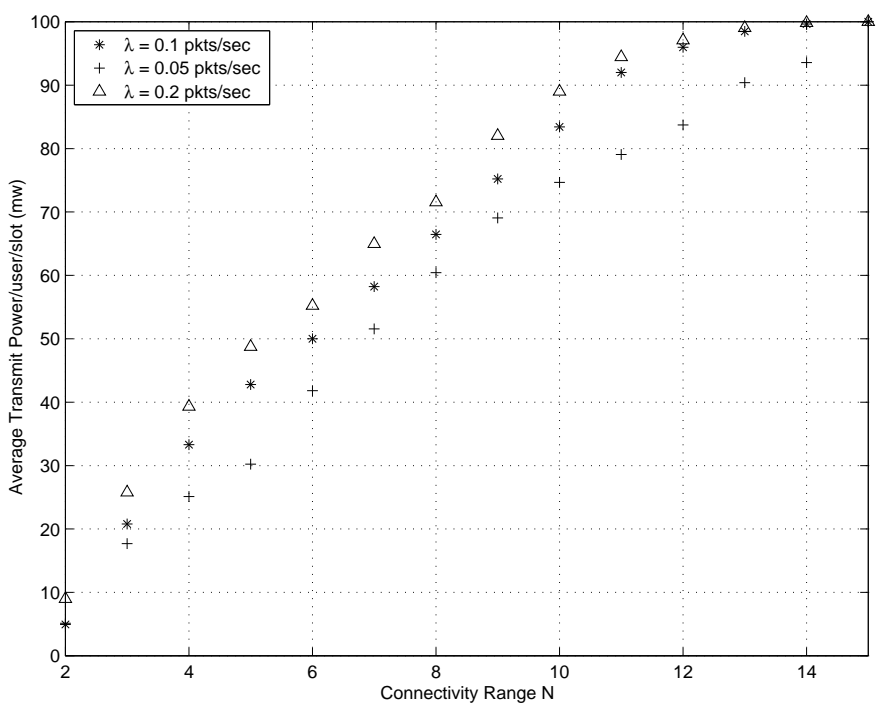

Figure 7: Average Power Consumption

to reduce the interference and hence improve the average node throughput as shown in Figure 8. Note the similarity of the average node throughput trends under both approaches of power management. Figure 9 shows the

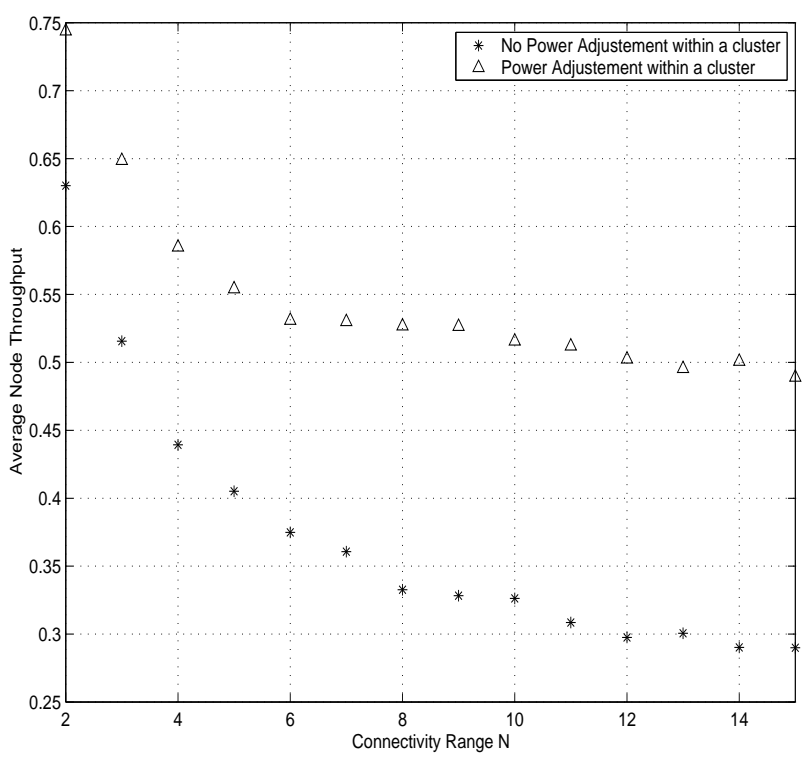

Figure 8: Average Node Throughput

end-to-end network throughput under the two proposed approaches for power management. It is evident that the trends are the same for both approaches. Moreover, it is noticed that in the "power adjustment within a cluster" approach, the maximum throughput is achieved at a different connectivity range, i.e., at $N=4$. In addition, at higher connectivity ranges, the "no power adjustment within a cluster" approach proves to be better. This is mainly due to the fact that in the approach which includes power adjustment within a cluster, minimum power routes has ten- 
dency to have more hops to the destination. This effect becomes more conspicuous for large values of $N$.

Finally, it is noticed from Figure 10 that the average power consumption has the same trends under the two power management approaches. The power consumption is lower when the second approach is used, since direct neighbors communicate using the minimum power needed for reliable communication. Hence, it can be concluded that the second power management approach outperforms the first one in terms of power savings and end-to-end throughput as well.

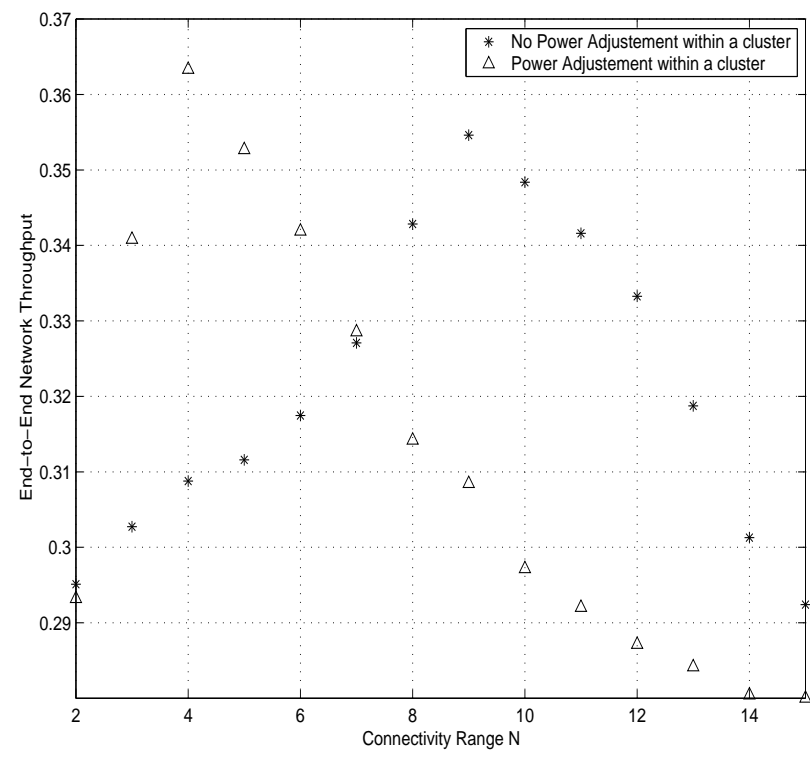

Figure 9: End-to-End Network Throughput

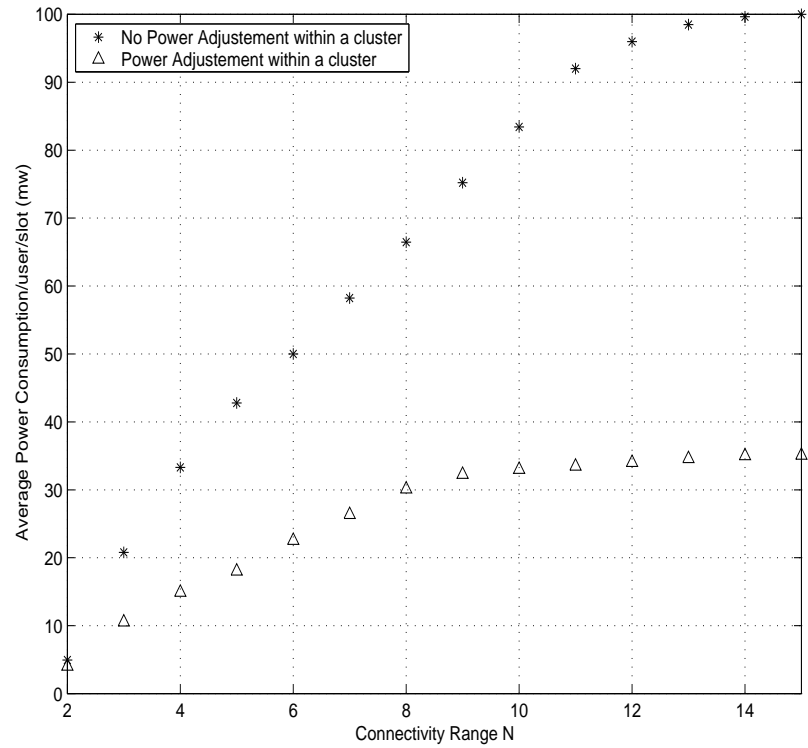

Figure 10: Average Power Consumption

\section{Possible Protocol Implementations}

In this section, we propose two protocols that enable each node to dynamically adapt the connectivity range parameter $N$ in order to achieve a near-optimal operating point. This is motivated by the fact that the optimal connectivity range changes with the dynamics of the network configuration characterized by the topology, nodes' mobility, and traffic load.

\section{A. Periodic Update Protocol (PUP)}

This protocol follows the following steps:

1. Initially, each node independently chooses its connectivity range to be the minimum i.e., the range $N$ is set to 2 .

2 . The node operates for a pre-specified number of frames (k) with this chosen value of $N$.

3. By the end of this period (called the checkpoint), the performance measure, namely the end-to-end throughput of this node is computed.

4. At this checkpoint, each node broadcasts its end-to-end throughput on the afore mentioned reverse channel (This is essential for each node to compute the average end-toend network throughput). This value is then stored in a data structure denoted by $\eta_{N}$.

5. The connectivity range is then increased by one, i.e. $N$ is increased by one. The ad-hoc system is expected to operate using this connectivity range for the next $k$ frames.

6. At the next checkpoint, the new value of the average end-to-end network throughput is computed and stored in a data structure denoted $\eta_{N}$.

7. Compare $\eta_{N-1}$ to $\eta_{N}$. One of the following two cases might arise:

If $\left(\eta_{N}>\eta_{N-1}\right)$

\{

Increase the connectivity range to $N+1$ and go to step 6 . \}

else

\{

Reduce the connectivity range to $\mathrm{N}-1$ and go to step 6 . \}

8. As long as the average end-to-end network throughput increases with $N$, we keep increasing $N$. This is done until the throughput starts decreasing with $N$, at some value of $N$, say at $N=i+1$. This implies that a maximum in the throughput is achieved at $N=i$, which is the connectivity range that achieves the maximum end-to-end network throughput for the current network configuration.

9. At each check point, compare the end-to-end network throughput with the throughputs achieved when $N=i+1$ and $N=i-1$. As long as $\eta_{i}>\eta_{i+1}$ and $\eta_{i}>\eta_{i-1}, N$ need not be changed. 
10. If the network configuration changes at some later time, such that this connectivity range $N=i$ does not achieve the maximum throughput any more, then pick any of the two neighboring points, $\eta_{i+1}$ or $\eta_{i-1}$, that achieves a higher throughput.

11. If $N=i+1$ achieves a higher throughput, then we further increase $N$ in order to search for the new maximum. Go to step 6.

12. If $N=i-1$ achieves a higher throughput, then we further decrease $N$ in order to search for the new maximum. Go to step 6.

\section{B. Quasi-Periodic Update Protocol (QPUP)}

This protocol is identical to the Periodic Update Protocol except that, when the network achieves maximum end-toend throughput, the algorithm less frequently attempts to test if the current connectivity range is the optimal. The algorithm takes advantage of the fact that the network under consideration consists of nodes of low mobility, i.e. the network topology changes slowly. Therefore, once the system reaches an operating point wherein the throughput is maximum, the algorithm expects the throughput to stay at the maximum or at a value very close to the maximum until the topology changes drastically. Therefore, this algorithm trades simplicity for performance. It is much simpler than the Periodic Update protocol, but there is a possible degradation in the end-to-end network throughput.

\section{CONCLUSIONS}

In this paper we have introduced the notion of power management within the context of wireless ad-hoc networks. The objective was to investigate the impact of using different transmit powers on the average power consumption, and the percentage of packets successfully reaching destinations, which we define as end-to-end network throughput. We defined the concept of clusters wherein a node adapts its transmit power so as to establish connectivity with only a limited number of neighborhood nodes. Within its cluster the power might wish to adapt power to communicate with different nodes, or it might use the same power to communicate with all nodes within the cluster. We found that the former scheme performs better in terms of achieving a lower average power consumption and a higher end to end throughput. Simulations further show that both schemes help improve performance in terms of average power consumption and end to end throughput. Thus, a network with a power management scheme implemented will have better performance than a network without such a scheme. A possible extension for this work is to consider the more general case where each mobile node has a different connectivity range. It gives more degrees of freedom to the network designer and is expected to improve throughput.

\section{REFERENCES}

[1] N. Bambos "Toward Power-sensitive Network Architectures in Wireless Communications: Concepts, Issues, and Design Aspects", IEEE Personal Communications Magazine, pp. 50-59, June 1998.

[2] J. Broch et al., "A Performance Comparison of Multi-Hop Wireless Ad-Hoc Network Routing Protocols", Proceedings of the ACM/IEEE MOBICOM'98.

[3] M. Corson and A. Ephremides, "A Distributed Routing Algorithm For Mobile Wireless Networks", Wireless Networks, Vol. 1, pp. 61-81, 1995.

[4] D. Bertsekas and R. Gallager, "Data Networks", Prentice-Hall Inc., 1987 (2nd Ed. 1992).

[5] M. Steenstrup, "Routing in Communications Networks", Prentice-Hall, Inc. 1995.

[6] G. Foschini and Z. Miljanic, "A Simple Distributed Autonomous Power Control Algorithm and its Convergence", IEEE Transactions on Vehicular Technology, Vol. 42, No. 4, Nov. 1993. 